\title{
Review Article \\ Serological Diagnosis and Follow-Up of Human Cystic Echinococcosis: A New Hope for the Future?
}

\author{
Raúl Manzano-Román, ${ }^{1}$ Carlos Sánchez-Ovejero, ${ }^{1}$ Ana Hernández-González, \\ Adriano Casulli, ${ }^{3}$ and Mar Siles-Lucas ${ }^{1}$ \\ ${ }^{1}$ Instituto de Recursos Naturales y Agrobiología de Salamanca (IRNASA-CSIC), Cordel de Merinas 40-52, 37008 Salamanca, Spain \\ ${ }^{2}$ Instituto de Salud Carlos III, Centro Nacional de Microbiología, Majadahonda, 28220 Madrid, Spain \\ ${ }^{3}$ Istituto Superiore di Sanità, Viale Regina Elena 299, 00161 Rome, Italy
}

Correspondence should be addressed to Adriano Casulli; adriano.casulli@iss.it

Received 13 April 2015; Accepted 30 August 2015

Academic Editor: Malcolm Jones

Copyright ( 2015 Raúl Manzano-Román et al. This is an open access article distributed under the Creative Commons Attribution License, which permits unrestricted use, distribution, and reproduction in any medium, provided the original work is properly cited.

Cystic echinococcosis (CE) is an important helminthic zoonotic disease caused by the Echinococcus granulosus complex. In humans, $\mathrm{CE}$ is a chronic disease driven by the growth of echinococcal cysts in different organs. Prognosis of this disease depends on multiple factors, including location, number, size, and stage of the cysts, making CE a disease of complex management. CE is usually asymptomatic for years and attracts limited attention from funding organizations and health authorities. For this reason, only experts' recommendations are available but no evidence-based conclusions have been drawn for CE clinical management. One of those pitfalls refers to the lack of evidence to support the use of serological tools for the diagnosis and follow-up of CE patients. In this respect, crude antigens are used to detect specific antibodies in patients, giving rise to false positive results. The advent of molecular techniques allowing the production of recombinant proteins has provided a number of candidate antigens that could overcome the problems associated with the use of crude parasite extracts in the serological assays. In this review, we present the last advances in this field, proposing the use of serology to support cyst stage-specific diagnosis and follow-up.

\section{Introduction}

Cystic echinococcosis (CE) is a parasitic disease caused by the larval stage (metacestode) of Echinococcus granulosus complex which affects livestock, wildlife, and humans. CE has a worldwide geographic distribution, remaining highly endemic in many pastoral communities, including several European countries [1]. CE global prevalence is estimated at 2-3 million human cases and a burden of 1 million DALYs accounting for underreporting [2]. In humans, CE is a chronic disease characterized by the long term growth of hydatid cysts in internal organs, mainly liver and lungs, with a complex clinical management. CE results in severe and life-threatening complications, with estimated mortality rates of $2-4 \%$ per 100.000 inhabitants [3, 4]. Many CE cases are asymptomatic for years and its diagnosis is still challenging due to the absence of pathognomonic signs. For this reason $\mathrm{CE}$ is frequently underdiagnosed and detected only when complications arise or by chance. Additionally, the clinical management of CE (i.e., surgery, percutaneous treatment, and/or chemotherapy) has many associated risks for relapses, pointing out the importance of the follow-up of patients.

CE diagnosis and monitoring firstly rely on imaging techniques. Ultrasonography (US) standardized classification of stage-specific cystic images has been issued by the WHO Informal Working Group on Echinococcosis (WHO-IWGE) for the diagnosis and the clinical management of CE [5]. Effective serological tests for CE diagnosis would be of great help to define and support cyst status and their evolution (active: CE1, CE2, and CE3b, transitional: CE3a, or inactive: CE4 and CE5) $[5,6]$. The main serological methods used for human CE diagnosis and follow-up are based on the detection of specific IgG antibodies. In this context, a number of drawbacks have been detected, including low sensitivity/specificity (Se/Sp) and a poor prognostic value for follow-up due to the long-lasting persistence of antibodies 
against hydatid fluid (HF) [7]. These pitfalls lead clinicians to consider serology against $\mathrm{HF}$ as an approach of little value, with doubtful benefit for the clinical management of CE. Alternative methods based on the detection of other antibody isotypes and IgG subisotypes against HF have been published [8]. Additionally, many authors have focused their research both on recombinant proteins and on synthetic peptides, to develop more sensitive and specific tests. Numerous recombinant proteins $(\mathrm{Rec})$ and related peptides, mainly derived from the antigen $\mathrm{B}$ and antigen 5 , have been tested for the detection and follow-up of antibodies in correlation with US findings. Unfortunately, available data were generated from small and underpowered clinical studies that have showed dissimilar Se and Sp for the same recombinant antigen [9]. Nevertheless, there are hints showing that some antigens are differentially expressed in different cyst stages, and thus antibody levels against these antigens could be associated with cyst activity and posttreatment outcome (i.e., surgery or chemotherapy) and could be applied for diagnosis and follow-up of CE patients $[9,10]$. In this context, a better characterization and standardization of each antigen should be performed to clearly define its role within CE serology.

In this paper we summarize the current knowledge on the use of HF for human CE diagnosis. Additionally, results obtained from different purified fractions of parasite antigens, recombinant antigens, and synthetic peptides are also revised. A comprehensive review of the different available antigens and their performance in the diagnosis of CE was published by Carmena and colleagues [11]. In this review, we also update the findings about the available serological tools from 2006 to date.

\section{Hydatid Fluid}

HF is a complex mixture of parasite-derived proteins, mainly produced by the germinal layer of the cyst. Some of the HF components have been characterized as highly immunogenic, reaching the host environment and triggering antibody responses. The HF is the main antigenic component in the majority of commercially and in-house serological assays. This antigen mixture is used in several techniques such as the enzyme linked immunosorbent assay (ELISA), the indirect haemagglutination test (IHA), and the immunoblotting (IB). Both the ELISA and the IHA are usually the first line tests for CE patients, while the IB is used as confirmatory test. As mentioned, the use of HF for the detection of CE specific antibodies is limited by several drawbacks. First, a percentage of CE patients are serological negative against HF. Specifically, the use of HF for the detection of total IgG in ELISA test leads to variable results regarding Se and Sp. In Table 1, a number of recent studies that used IgG-ELISA tests for CE diagnosis are shown. Se reported in these studies varied from $64.8 \%$ to $100 \%$. Reasons for false negative results depend on several factors comprising cyst location other than the liver $[12,13]$, early (CE1) and inactive (CE4 and CE5) cyst stages [14-16], serum collection before treatment $[15,17]$, single and small cysts [17], and HF antigenic source variability [18]. Additionally, E. granulosus complex comprises several genotypes that potentially express different antigenic sets. For instance, it has been shown that the G1 and G2 genotypes from Europe, contrary to those from China, express high quantities of antigen B2 in HF [19]. This may lead to different diagnostic performance of a specific HF. The differential expression of antigens is not only a qualitative matter, but also quantitative, depending on cyst stages. Recently, a proteomic and immunoproteomic study has shown that CE1 and CE2 cyst stages differ in the expression of their immunodominant antigens (antigen B and antigen 5). Antigen 5 is predominant and recognized by antibodies from patients with early cyst stages, while antigen B is the most scarce in CE1 cyst stage and mainly detected in patients with CE2 and CE3 cyst stages [20]. These differences might be useful in clinical practice to correctly define cyst stages and their viability by using the most indicated antigen for a stage-specific diagnosis. A second problem using HF is the percentage of false positive results detected. For instance, IgG-ELISA tests based on HF as antigen give rise to low false positive results in healthy donors (e.g., Sp from $87.5 \%$ in Indian donors to $100 \%$ in Italian donors) $[15,21]$. Cross-reactivity is quite high in patients with other parasitic diseases, such as alveolar echinococcosis (AE) and cysticercosis, but also schistosomiasis and fascioliasis $[22,23]$. For instance, the cross-reactivity of E. granulosus $\mathrm{HF}$ with antibodies from AE patients can reach more than $50 \%$ [17]. The HF has also been shown not to be a good antigen for patients' follow-up during the clinical management of CE. During the follow-up, ELISA-IgG test is difficult to interpret [24], and anti-HF IgG antibody reactivity may remain high many years after successful cyst removal [25].

The detection of antibodies other than $\operatorname{IgG}$ has shown some promising results in relation to cyst activity, relapses, and follow-up. It has been shown that both IgG2 and IgG4 could be related to cyst stages, disease evolution, and relapses $[9,26,27]$. Remarkably, it is known that the subisotype responses against $\mathrm{CE} 1, \mathrm{CE} 2$, and $\mathrm{CE} 3$ cyst stages are mainly IgG4, while IgG1, IgG2, and IgG3 responses predominate against CE4 and CE5 cysts, although this is still a question of debate [11,22]. Antibody isotypes different than IgG can be also detected against $\mathrm{HF}$ in CE patients. IgE and $\operatorname{IgM}$ antibodies have been considered as better markers than IgG after chemotherapy and surgery [28]. Nevertheless, these isotypes are more frequently underdetected in CE patients, similar to different IgG subisotypes [29-31].

\section{Antigens Derived from Hydatid Fluid}

3.1. Antigen B. In an attempt to overcome the problems related to the use of $\mathrm{HF}$ for the detection of antibodies in $\mathrm{CE}$ patients, many authors have described the production and the use in serological tests of partially purified native antigens, recombinant antigens, and synthetic peptides. These are mainly represented by the two most immunogenic antigens in HF: antigen B (EgAgB) and antigen 5 (EgAg5).

$\mathrm{EgAgB}$ is a polymeric protein of $120-160 \mathrm{kDa}$ that dissociates under reducing conditions in 8,16 , and $20-24 \mathrm{kDa}$ subunits. Its biological role includes the protease inhibitor activity, neutrophil chemotaxis inhibition, triggering of nonprotective Th2 responses, induction of apoptosis of immune cells, and sequestration of xenobiotics [22]. EgAgB is codified 
TAble 1: Performance of the hydatid cyst fluid in ELISA test for the detection of total IgG in CE patients (articles published from 2006).

\begin{tabular}{|c|c|c|c|c|}
\hline Number of CE patients & Confirmatory test & Sensitivity (\%) & Negative serology more frequent when & Reference \\
\hline 23 & Histopathology & 100 & Not specified & \multirow{3}{*}[40]{} \\
\hline 5 & Imaging techniques plus serology & 80 & Not specified & \\
\hline 13 & Serology & 100 & Not specified & \\
\hline 41 & Surgery & 95.1 & Not specified & {$[21]$} \\
\hline 6 & Imaging techniques & 66.7 & Cyst location other than liver & {$[12]$} \\
\hline 144 & Imaging techniques & 92.4 & CE1, CE4, and CE5 cyst stages & {$[14]$} \\
\hline 123 & Imaging techniques & $64.8^{*}$ & CE4 and CE5 cyst stages, no pretreatment & [15] \\
\hline 59 & Surgery & 95.1 & Not specified & {$[20]$} \\
\hline 10 & Surgery & 100 & Not specified & {$[52]$} \\
\hline 54 & Surgery & 81.5 & \multirow{2}{*}{ Single cyst, no pretreatment } & \multirow{2}{*}[17]{} \\
\hline 186 & Surgery & 83.3 & & \\
\hline 32 & Imaging techniques & 93.8 & CE4 and CE5 cyst stages & {$[16]$} \\
\hline 155 & Surgery & $90.3^{*}$ & Not specified & [29] \\
\hline 40 & Surgery & 92.5 & Not specified & {$[30]$} \\
\hline \multirow{7}{*}{47} & \multirow{7}{*}{ Surgery } & $95.7^{1}$ & \multirow{7}{*}{$\begin{array}{l}\text { HF collected from cysts of different hosts } \\
\text { or of different anatomical locations from } \\
\text { the same host }\end{array}$} & \multirow{7}{*}[18]{} \\
\hline & & $93.6^{2}$ & & \\
\hline & & $91.4^{3}$ & & \\
\hline & & $97.8^{4}$ & & \\
\hline & & $93.6^{5}$ & & \\
\hline & & $78.5^{6}$ & & \\
\hline & & $72.2^{7}$ & & \\
\hline \multirow{2}{*}{63} & \multirow{2}{*}{ Surgery } & 90.5 & \multirow{2}{*}{ Not specified } & \multirow{2}{*}[48]{} \\
\hline & & $82.4^{*}$ & & \\
\hline 68 & Imaging techniques & 92.6 & Lung cysts & {$[13]$} \\
\hline
\end{tabular}

${ }^{*}$ Commercial test; HF of cysts from ${ }^{1}$ sheep liver, ${ }^{2}$ sheep lungs, ${ }^{3}$ goat liver, ${ }^{4}$ human liver, ${ }^{5}$ camel lungs, ${ }^{6}$ cow lungs, and ${ }^{7}$ cocktail.

by a multigenic family, with at least five genetic groups: EgAgB1, EgAgB2, EgAgB3, EgAgB4, and EgAgB5 [32-35]. These different subunits share from $44 \%$ to $81 \%$ of sequence identity. Each isoform has more than $90 \%$ homology with Echinococcus granulosus and E. multilocularis species, and although lower, similar antigens are also found in the genus Taenia [11]. These homologies can give rise to cross-reactivity with other helminthic parasites. EgAgB has been obtained as a native purified antigen, in its recombinant form. The purified native protein has a Se ranging from $60 \%$ to $85 \%$ in ELISA test and from $60 \%$ to $92 \%$ in immunoblot [11]. From 2006 up to date, several publications showed a high variability in the Se of this purified antigen, ranging from $54 \%$ to $100 \%$ (Table 2). Different cyst stages and their followup in US may reflect different profiles of antigens produced and released by the host immune system [20]. For instance, $\mathrm{EgAgB}$ recombinant antigen, in ELISA test, showed a Se of $74 \%, 96 \%, 90 \%$, and $56 \%$ in patients with CE1, CE2, CE3, and CE4/CE5 cyst stages, respectively [36]. Additional reasons for false negative results have been pointed out by several authors, including cyst localization other than liver and small size of cysts (see Table 2). These results are similar to those found for false negative reactions against HF. The heterogeneity and thus the limited value of native antigens are also found when the purified $\mathrm{EgAgB}$ is used for the serodiagnosis of CE patients. In this respect, antigens purified from different sources of HF result in a variable Se in the same patients' cohort (from $82.1 \%$ to $96.9 \%$; [37]; Table 2).

In its recombinant form (Rec), mainly two isoforms have been assayed: RecEgAgB1 and RecEgAgB2. RecEgAgB1 gives rise to variable sensitivities, ranging from $55 \%$ to $84 \%$ [11] and from $71 \%$ to $94.6 \%$ in subsequent studies [38] (Table 2). The lowest Se (71\%; [38]) was attributed to false negative results due to patients with Echinococcus genotypes other than G1. Nevertheless, additional factors such as the presence of CE4 and CE5 cyst stages and serum sampling collection with respect to treatment are probably contributing to false negative results against this recombinant antigen [15]. Some of these factors have been also pointed out for RecEgAgB2, for which false negatives have been related to single cysts and to sera collected before treatment with benzimidazoles [17]. RecEgAgB2 has given rise to very variable sensitivities as well ([11]; Table 2). A modified version of the RecEgAgB2, consisting of twofold tandem repeat of the original recombinant protein, showed a Se similar to the single antigen unit [17]. Additional isoforms of $\mathrm{EgAgB}$ have been tested on few occasions (Table 2). RecEgAgB3 and B5 did not improve the Se of other antigenic isoforms [19], but RecEgAgB4 has shown promising results (Se from $75.8 \%$ to $91.7 \%$ ) [19, 39]. Remarkably, EgAgB3 and B5 were not found after a proteomic 


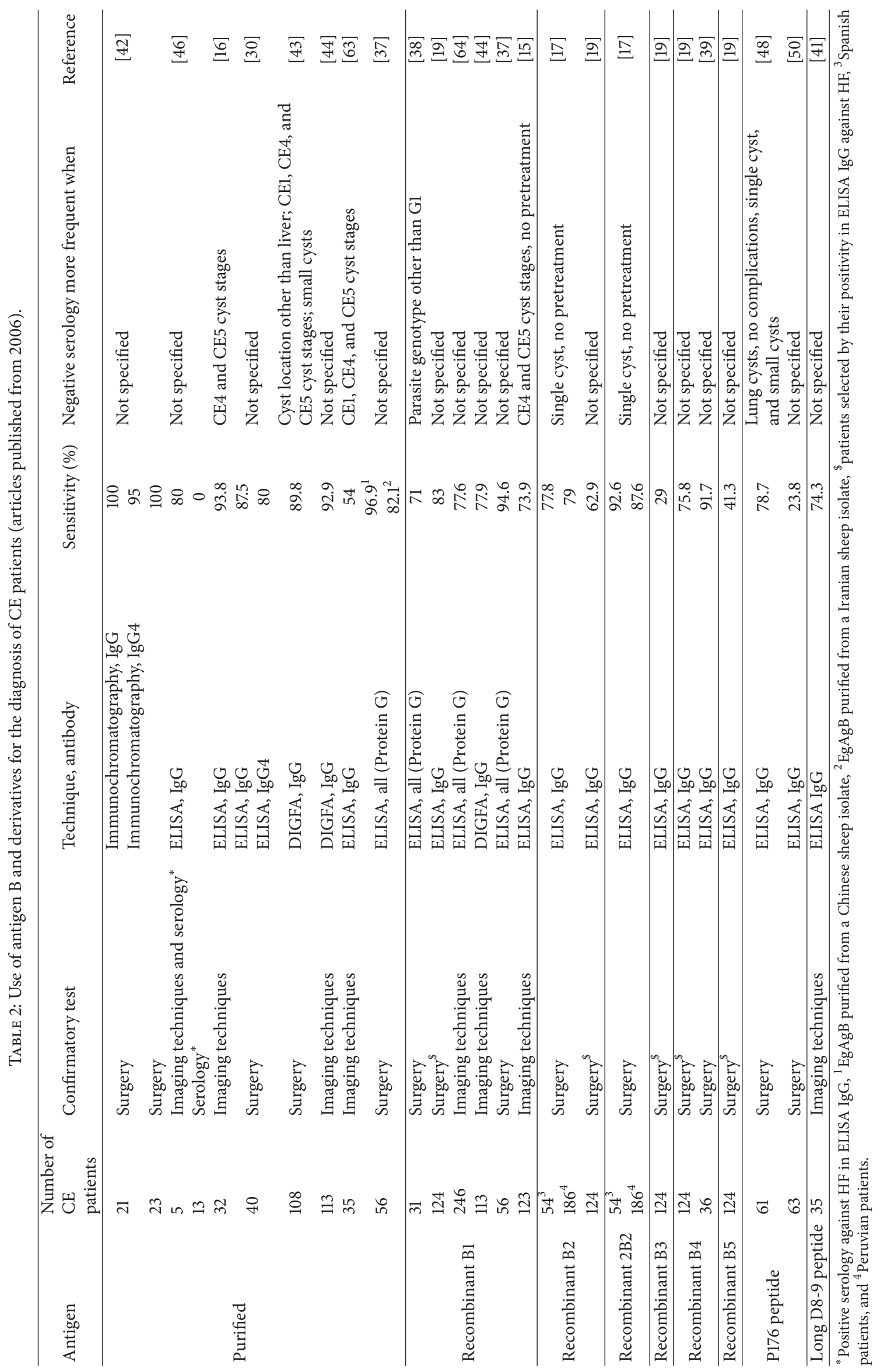


study on the composition of the HF in CE1 and CE2 cyst stages from sheep [40].

As mentioned, several synthetic peptides derived from EgAgB1 have been also tested in ELISA for the detection of specific antibodies. From those tests, the most promising results have been obtained with the p176 peptide ([11]; Table 2). Nevertheless, the diagnostic performance of synthetic peptides has been worse than that of the whole original molecule, either in its purified native or in recombinant forms. In addition, pooling three peptides has not resulted in the improvement of the test Se [41]. In any case, the use of bioinformatics for peptides selection and their screening in high-throughput platforms have been stated as a proof of principle and this approach could be used in the future for the identification of relevant diagnostic epitopes. As mentioned, a dominant IgG4 response is usually associated with active and transitional cysts, whereas inactive cysts mainly trigger IgG1, IgG2, and IgG3 responses. This has important implications in the interpretation of the usefulness of the detection of different IgG isotypes, since the level of reactivity of each subisotype could be driven by the cyst stages found in patients. This is the case for IgG4, since the Se obtained when detecting this subisotype is lower than that detected when total specific IgG levels are evaluated [42]. Whether this lower Se is associated with inactive cysts, it should be further evaluated to ascertain the usefulness of IgG4 instead of total IgG, both for the diagnosis and for the follow-up of CE patients. The Sp of EgAgB in its different isoforms is also variable, although the main cross-reactivity has been reported with patients affected by AE and cysticercosis [11]. Additionally, antibodies from patients with other parasitic diseases including onchocerciasis, schistosomiasis, and toxocariasis have given rise to false positive reactions when tested against EgAgB [11]. It is worth mentioning the efforts of several authors to test new affordable and easy to use serological techniques instead of ELISA or the IB tests, making those immunoassays stand out based on particles such as DIGFA (dot immunogold filtration assay [43, 44]; Table 2).

In the light of the results obtained by several authors using EgAgB, this antigenic family looks promising for the serodiagnosis of $\mathrm{CE}$ patients and potentially useful in the follow-up. Anyway, testing of this antigen family obtained in a standardized format, with a well-defined and wide panel of CE samples, including the clinical information influencing the interpretation of the test (the parasite genotype, location, number, size, and type of cysts, and presence of complications and coinfections), should be further performed.

3.2. Antigen 5. Antigen 5 (EgAg5) is also an abundant highly immunogenic component of HF. EgAg5 is a thermolabile protein of around $400 \mathrm{kDa}$, composed of two subunits of 57 and $67 \mathrm{kDa}$. Studies on the $\mathrm{N}$-terminal sequence of the $38 \mathrm{kDa}$ subunit have shown that several isoforms exist similar to $\mathrm{EgAgB}$, and thus EgAg5 could be also coded by a multigenic family [45]. The biological function of EgAg5 is largely unknown, and although the $38 \mathrm{kDa}$ subunit shows homologies with trypsin peptidases, the enzymatic activity is lacking [46]. Additionally, the smallest subunit of $22 \mathrm{kDa}$ contains proteoglycans of the heparan sulfate group and calcium-binding motifs, suggesting that this subunit could interact with the extracellular matrix and the cell surface [47].

Molecules very similar to EgAg5 (more than 90\% identity) are also expressed by E. multilocularis and the genus Taenia; thus cross-reactivity of this antigen with antibodies from patients affected by AE and cysticercosis is expected. As we explain in the following paragraphs, the diagnostic use of EgAg5 has shown mainly drawbacks regarding Se and Sp. The purified native EgAg5 has been obtained using different methodologies, including size exclusion chromatography and immunoaffinity. Lately, an easy and efficient method for the enrichment of this antigen from HF has been described [48]. Variability of its performance in serodiagnosis could be also attributed to differences in the clinical status of the patients. For instance, EgAg5 seems to play a role in the induction of specific antibodies mainly when CE1 and CE5 cyst stages are present [40]. This variable Se has been reported by several authors, ranging from $50 \%$ to $87.5 \%$ [11, 49]. As recombinant proteins, EgAg5 has been tested in its full format (RecEgAg5) and in its $38 \mathrm{kDa}$ version (RecEgAg53.38) by Auer and colleagues [50]. Although the $38 \mathrm{kDa}$ subunit has been described as the most antigenic component detected by IB, the Se of the corresponding recombinant antigen was very low $(21 \%)[50,51]$. Similarly, low and variable sensitivities (from $16 \%$ to $85 \%$ ) have been detected using the p89-122 EgAg5 peptide [11]. Due to the low Se of EgAg5 and derivatives, this antigen has been less frequently tested than EgAgB. Nevertheless, reasons for this low Se could be due to cyst stage; thus the usefulness of this antigen for the detection of early active and late inactive cyst lesions should be further evaluated.

3.3. Other Antigens. The most frequently used crude extract of E. granulosus for the serodiagnosis of $\mathrm{CE}$ has been the somatic extract of protoscoleces (EgPpsSom). This extract has a Se ranging from $69.4 \%$ to $96.9 \%$ [11, 16, 43, 44, 52-54]. When compared with HF, the EgPpsSom performs worse. Less frequently, other somatic extracts have been applied in the serodiagnosis of CE patients such as those derived from the cyst wall (96.7\% Se; [52]), the tegument of protoscoleces (81.3\% Se; [16]), and the adult worm (82\% Se; [55]). Crossreactivity of those extracts is similar to that detected in HF. Drawbacks associated with the heterogeneity of somatic extracts could be attributed to these antigenic preparations. In addition, reasons for the presence of false negative results are similar to those found when the HF is used as antigenic source (cyst location other than liver, small size cysts, and CE1, CE4, and CE5 cyst stages).

Additionally, several recombinant antigens different from $\mathrm{EgAgB}$ and EgAg5 have been obtained by several authors and tested for the diagnosis of CE. These include, among others, the malate dehydrogenase (RecEgMDH), the calcium binding protein ( $\mathrm{RecEgCaBP})$, the actin filament fragmenting protein (RecEgAFFP), the RecEgEpCl, the thioredoxin peroxidase (RecEGTPx), and the RecEg19 [11, 56, 57]. RecEGTPx and RecEg19 showed low Se $(<45 \%$; [56-58]). The rest of the above-mentioned antigens showed variable Se (even for the same recombinant) ranging from $45 \%(\mathrm{MDH})$ to $90 \%$ 
(MDH, EpC1) and variable Sp (even for the same antigen) ranging from $83 \%$ to $95 \%$ [11]. For this reason these antigens could be considered as good alternatives for serological tests, although further characterization is needed to evaluate their diagnostic potential.

\section{Antigens for Clinical Management}

The clinical management of CE should be based on recommendations done by experts and driven by cystic stages identification [54]. In this context, the WHO-IWGE has proposed a cyst classification based on active, transitional, and inactive cysts.

Some antigens could be mainly expressed by defined cyst stages, showing that serology could be potentially useful for the definition of cyst activity and thus for the clinical management of CE patients. As mentioned, the HF has been also evaluated for the follow-up of patients treated by surgery and/or chemotherapy, but its usefulness is hampered by the long persistence of antibodies in patients with nonactive cysts [59]. A better correlation of specific antibodies against defined active cyst stages has been reported in patients after surgical treatment, including EgAgB [59, 60], EgAgP29 [60], and the heat-shock protein 20 [61]. Several authors have also found a correlation between a low level of specific antibodies against RecEgAgB2, RecEgAgHSP20, and RecEgAgB1 and the presence of inactive cysts $[36,59,61]$. Nevertheless, some of those antigens are not recognized by a percentage of CE patients [60]. Additionally, it has been shown that the banding pattern in IB from CE patients changes depending on the cyst stage [62].

Similarly, other authors have detected the loss of defined bands in IB after cure. The identification and characterization of the above-mentioned antigens could support the clinical decision making actually based on imaging techniques. Similarly, the shift of defined subisotypes of IgG antibodies during cyst evolution could be used for the follow-up of CE patients. As mentioned, IgG4 levels could be correlated with cyst activity, and declining of the levels of isotypes other than IgG seems to be useful to define the success of treatment $[26,27]$. Nevertheless, detectable levels of specific IgG4 and especially of $\operatorname{IgE}$ and $\operatorname{IgM}$ are only found in a relative small percentage of CE patients.

\section{Conclusions: A New Hope for the Future?}

Diagnosis and follow-up of CE patients are mainly based on imaging techniques. These can be used for the identification of cystic stages, leading to a stage-specific approach in $\mathrm{CE}$ clinical management. Serological tools supporting imaging techniques would be desirable. However the available tests are based on antibodies against crude antigens and thus marred by poor Sp and Se, with low or no usefulness for the followup of patients during the treatment. Specific recombinant antigens have good potential as diagnostic and follow-up tools for CE, but progress in this field is hampered by lack of standardization. Thus, a challenge still exists to develop a reliable world standard based on serology for the diagnosis and monitoring of CE patients. In this respect, a multicentre study with a wide panel of sera from CE patients, including relevant clinical data to properly define the usefulness of the available recombinant antigens, should be performed. Along this path, a project focusing on $\mathrm{CE}$ has been recently funded by the European commission under the Seventh Framework Programme (HERACLES; http://www.heracles-fp7.eu/). In the framework of HERACLES, six recombinant antigens (RecEgAgB1, RecEgAgB2, RecEgAg5, RecEgAgMDH, RecEgAgCaBP, and RecEgAgAFFP) will be tested for the serodiagnosis and follow-up of $\mathrm{CE}$ on a wide panel of samples obtained from extended ultrasound surveys in Eastern Europe. These recombinant antigens have been already produced in a standardized way and preliminary tested in ELISA for the detection of specific IgG in sera. Additionally, two databases for the collection of clinical data from retrospective/prospective patients providing serum samples have been developed (the database behind the European Register of Cystic Echinococcosis ERCE, http://www.heracles-fp7.eu/erce.html, and CYSTRACK database, http://cystrack.irnasa.csic.es). Hosting of samples and clinical data has been organized in a dedicated biobank (EchinoBiobank). This strategy will hopefully pave the way to improve the diagnosis and follow-up of CE patients, providing evidence-based data on the usefulness of serology in CE clinical management.

\section{Disclosure}

The funding sources had no involvement in the preparation, ideas, writing, interpretation, or the decision to submit this paper.

\section{Conflict of Interests}

The authors declare that there is no conflict of interests regarding the publication of this paper.

\section{Acknowledgments}

The research that will lead to these results has received funding from the European Community's Seventh Framework Programme under the Grant agreement 602051 (Project HERACLES: Human cystic Echinococcosis ReseArch in CentraL and Eastern Societies; http://www.Heracles-fp7.eu/). The authors are grateful to Belgees Boufana for reviewing the text.

\section{References}

[1] G. Grosso, S. Gruttadauria, A. Biondi, S. Marventano, and A. Mistretta, "Worldwide epidemiology of liver hydatidosis including the Mediterranean area," World Journal of Gastroenterology, vol. 18, no. 13, pp. 1425-1437, 2012.

[2] C. M. Budke, P. Deplazes, and P. R. Torgerson, "Global socioeconomic impact of cystic echinococcosis," Emerging Infectious Diseases, vol. 12, no. 2, pp. 296-303, 2006.

[3] T. Junghanss, A. M. da Silva, J. Horton, P. L. Chiodini, and E. Brunetti, "Clinical management of cystic echinococcosis: state 
of the art, problems, and perspectives," The American Journal of Tropical Medicine and Hygiene, vol. 79, no. 3, pp. 301-311, 2008.

[4] M. Belhassen-García, A. Romero-Alegria, V. Velasco-Tirado et al., "Study of hydatidosis-attributed mortality in endemic area," PLoS ONE, vol. 9, no. 3, Article ID e91342, 2014.

[5] E. Brunetti, P. Kern, and D. A. Vuitton, "Expert consensus for the diagnosis and treatment of cystic and alveolar echinococcosis in humans," Acta Tropica, vol. 114, no. 1, pp. 1-16, 2010.

[6] W. Hosch, T. Junghanss, M. Stojkovic et al., "Metabolic viability assessment of cystic echinococcosis using high-field ${ }^{1} \mathrm{H}$ MRS of cyst contents," NMR in Biomedicine, vol. 21, no. 7, pp. 734-754, 2008.

[7] T. S. Barnes, P. Deplazes, B. Gottstein et al., "Challenges for diagnosis and control of cystic hydatid disease," Acta Tropica, vol. 123, no. 1, pp. 1-7, 2012.

[8] R. K. Tenguria and M. I. Naik, "Evaluation of human cystic echinococcosis before and after surgery and chemotherapy by demonstration of antibodies in serum," Annals of Parasitology, vol. 60, no. 4, pp. 297-303, 2014.

[9] M. Benabid, Y. Galai, R. Nouira, S. Bouchoucha, A. Bouratbine, and K. Aoun, "Contribution of specific anti-hydatid IgG subclasses in the diagnosis of echinococcosis primary infection and relapses," Clinical Laboratory, vol. 59, no. 3-4, pp. 293-298, 2013.

[10] E. Ortona, P. Margutti, F. Delunardo et al., "Recombinant antigens of Echinococcus granulosus recognized by IgE and IgG4 of sera from patients with cystic echinococcosis," Parassitologia, vol. 46, no. 4, pp. 435-436, 2004.

[11] D. Carmena, A. Benito, and E. Eraso, "Antigens for the immunodiagnosis of Echinococcus granulosus infection: an update," Acta Tropica, vol. 98, no. 1, pp. 74-86, 2006.

[12] A. A. Kilimcioğlu, N. Girginkardeşler, M. Korkmaz et al., "A mass screening survey of cystic echinococcosis by ultrasonography, Western blotting, and ELISA among university students in Manisa, Turkey," Acta Tropica, vol. 128, no. 3, pp. 578-583, 2013.

[13] C. Akisu, S. B. Delibas, C. Bicmen, S. Ozkoc, U. Aksoy, and N. Turgay, "Comparative evaluation of western Blotting in hepatic and pulmonary cystic echinococcosis," Parasite, vol. 13, no. 4, pp. 321-326, 2006.

[14] J.-Y. Wang, C.-H. Gao, D. Steverding, X. Wang, F. Shi, and Y.-T. Yang, "Differential diagnosis of cystic and alveolar echinococcosis using an immunochromatographic test based on the detection of specific antibodies," Parasitology Research, vol. 112, no. 10, pp. 3627-3633, 2013.

[15] F. Tamarozzi, Y. Sako, A. Ito et al., "Recombinant AgB8/1 ELISA test vs. commercially available IgG ELISA test in the diagnosis of cystic echinococcosis," Parasite Immunology, vol. 35, no. 12, pp. 433-440, 2013.

[16] A. Schweiger, F. Grimm, I. Tanner et al., "Serological diagnosis of echinococcosis: the diagnostic potential of native antigens," Infection, vol. 40, no. 2, pp. 139-152, 2012.

[17] A. Hernández-González, S. Santivañez, H. H. García et al., "Improved serodiagnosis of cystic echinococcosis using the new recombinant 2B2t antigen," PLoS Neglected Tropical Diseases, vol. 6, no. 7, Article ID e1714, 2012.

[18] H. Rahimi, S. M. Sadjjadi, and B. Sarkari, "Performance of antigen $\mathrm{B}$ isolated from different hosts and cyst locations in diagnosis of cystic echinococcosis," Iranian Journal of Parasitology, vol. 6, no. 1, pp. 12-19, 2011.

[19] L. Jiang, Y.-G. Zhang, M.-X. Liu, and Z. Feng, "Analysis on the reactivity of five subunits of antigen $B$ family in serodiagnosis of echinococcosis," Experimental Parasitology, vol. 131, no. 1, pp. 85-91, 2012.

[20] X. Chen, J. Zhang, X. Feng et al., "Humoural immune response and pathological analysis in patients with false immune diagnosis of cystic echinococcosis," Parasite Immunology, vol. 36, no. 4, pp. 170-176, 2014.

[21] S. Chirag, B. A. Fomda, A. Khan et al., "Detection of hydatidspecific antibodies in the serum and urine for the diagnosis of cystic echinococcosis in patients from the Kashmir Valley, India," Journal of Helminthology, vol. 89, no. 2, pp. 232-237, 2015.

[22] W. Zhang, H. Wen, J. Li, R. Lin, and D. P. McManus, "Immunology and immunodiagnosis of cystic echinococcosis: an update," Clinical and Developmental Immunology, vol. 2012, Article ID 101895, 10 pages, 2012.

[23] P. Moro and P. M. Schantz, "Echinococcosis: a review," International Journal of Infectious Diseases, vol. 13, no. 2, pp. 125-133, 2009.

[24] C. Manterola, M. Vial, P. Schneeberger, J. L. Peña, J. Hinostroza, and A. Sanhueza, "Precision of ELISA-IgE and ELISA-IgG determination in the postoperative followup of patients with hepatic echinococcosis," Cirugia Espanola, vol. 81, no. 1, pp. 2327, 2007.

[25] Z. Galitza, E. Bazarsky, R. Sneier, J. Peiser, and J. El-On, "Repeated treatment of cystic echinococcosis in patients with a long-term immunological response after successful surgical cyst removal," Transactions of the Royal Society of Tropical Medicine and Hygiene, vol. 100, no. 2, pp. 126-133, 2006.

[26] S. D. Lawn, J. Bligh, P. S. Craig, and P. L. Chiodini, "Human cystic echinococcosis: evaluation of post-treatment serologic follow-up by IgG subclass antibody detection," American Journal of Tropical Medicine and Hygiene, vol. 70, no. 3, pp. 329-335, 2004.

[27] T. Celik, C. A. Akarsu, C. Gungor, C. Colak, and N. Ormeci, "Evaluation of antibodies against hydatid cyst fluid antigens in the post-treatment follow-up of cystic echinococcosis patients," Medical Science Monitor, vol. 15, no. 4, pp. CR170-CR176, 2009.

[28] M. P. Zarzosa, A. O. Domingo, P. Gutiérrez et al., "Evaluation of six serological tests in diagnosis and postoperative control of pulmonary hydatid disease patients," Diagnostic Microbiology and Infectious Disease, vol. 35, no. 4, pp. 255-262, 1999.

[29] I. Marinova, G. Nikolov, A. Michova, R. Kurdova, and B. Petrunov, "Quantitative assessment of serum-specific IgE in the diagnosis of human cystic echinococcosis," Parasite Immunology, vol. 33, no. 7, pp. 371-376, 2011.

[30] G. M. Tawfeek, H. S. Elwakil, L. El-Hoseiny et al., "Comparative analysis of the diagnostic performance of crude sheep hydatid cyst fluid, purified antigen B and its subunit (12 Kda), assessed by ELISA, in the diagnosis of human cystic echinococcosis," Parasitology Research, vol. 108, no. 2, pp. 371-376, 2011.

[31] E. Cappello, B. Cacopardo, E. Caltabiano et al., "Epidemiology and clinical features of cystic hydatidosis in Western Sicily: a ten-year review," World Journal of Gastroenterology, vol. 19, no. 48, pp. 9351-9358, 2013.

[32] V. Fernández, H. B. Ferreira, C. Fernández, A. Zaha, and A. Nieto, "Molecular characterisation of a novel 8-kDa subunit of Echinococcus granulosus antigen B," Molecular and Biochemical Parasitology, vol. 77, no. 2, pp. 247-250, 1996.

[33] G. Chemale, K. L. Haag, H. B. Ferreira, and A. Zaha, "Echinococcus granulosus antigen B is encoded by a gene family," Molecular and Biochemical Parasitology, vol. 116, no. 2, pp. 233-237, 2001.

[34] A. C. Arend, A. Zaha, F. J. Ayala, and K. L. Haag, "The Echinococcus granulosus antigen B shows a high degree of 
genetic variability," Experimental Parasitology, vol. 108, no. 1-2, pp. 76-80, 2004.

[35] K. L. Haag, L. Alves Jr., A. Zaha, and F. J. Ayala, "Contingent, non-neutral evolution in a multicellular parasite: natural selection and gene conversion in the Echinococcus granulosus antigen B gene family," Gene, vol. 333, pp. 157-167, 2004.

[36] T. Li, A. Ito, X. Chen et al., "Specific IgG responses to recombinant antigen B and Em18 in cystic and alveolar echinococcosis in China," Clinical and Vaccine Immunology, vol. 17, no. 3, pp. 470-475, 2010.

[37] T. Mohammadzadeh, Y. Sako, S. M. Sadjjadi, B. Sarkari, and A. Ito, "Comparison of the usefulness of hydatid cyst fluid, native antigen B and recombinant antigen B8/1 for serological diagnosis of cystic echinococcosis," Transactions of the Royal Society of Tropical Medicine and Hygiene, vol. 106, no. 6, pp. 371375, 2012.

[38] A. Ito, T. Dorjsuren, A. Davaasuren et al., "Cystic echinococcoses in Mongolia: molecular identification, serology and risk factors," PLoS Neglected Tropical Diseases, vol. 8, no. 6, Article ID e2937, 2014.

[39] E. Kalantari, M. Bandehpour, R. Pazoki et al., "Application of recombinant Echinococcus granulosus antigen B to ELISA kits for diagnosing hydatidosis," Parasitology Research, vol. 106, no. 4, pp. 847-851, 2010.

[40] C. S. Ahn, X. Han, Y. A. Bae et al., "Alteration of immunoproteome profile of Echinococcus granulosus hydatid fluid with progression of cystic echinococcosis," Parasites \& Vectors, vol. 8, no. 1, article 10, 2015.

[41] C. List, W. Qi, E. Maag, B. Gottstein, N. Müller, and I. Felger, "Serodiagnosis of Echinococcus spp. infection: explorative selection of diagnostic antigens by peptide microarray," PLoS Neglected Tropical Diseases, vol. 4, no. 8, article e771, 2010.

[42] A. Khalilpour, S. M. Sadjjadi, Z. K. Moghadam et al., "Lateral flow test using Echinococcus granulosus native antigen B and comparison of IgG and $\operatorname{IgG}_{4}$ dipsticks for detection of human Cystic echinococcosis," American Journal of Tropical Medicine and Hygiene, vol. 91, no. 5, pp. 994-999, 2014.

[43] X. Feng, H. Wen, Z. Zhang et al., "Dot immunogold filtration assay (DIGFA) with multiple native antigens for rapid serodiagnosis of human cystic and alveolar echinococcosis," Acta Tropica, vol. 113, no. 2, pp. 114-120, 2010.

[44] X. Chen, X. Chen, X. Lu, X. Feng, and H. Wen, "The production and comparative evaluation of native and recombinant antigens for the fast serodiagnosis of cystic echinococcosis with dot immunogold filtration assay," Parasite Immunology, vol. 37, no. 1, pp. 10-15, 2015.

[45] L.-H. Zhang and D. P. McManus, "Purification and N-terminal amino acid sequencing of Echinococcus granulosus antigen 5," Parasite Immunology, vol. 18, no. 12, pp. 597-606, 1996.

[46] C. Lorenzo, G. Salinas, A. Brugnini, C. Wernstedt, U. Hellman, and G. González-Sapienza, "Echinococcus granulosus antigen 5 is closely related to proteases of the trypsin family," Biochemical Journal, vol. 369, no. 1, pp. 191-198, 2003.

[47] A. Siracusano, F. Delunardo, A. Teggi, and E. Ortona, "Hostparasite relationship in cystic echinococcosis: an evolving story," Clinical and Developmental Immunology, vol. 2012, Article ID 639362, 12 pages, 2012.

[48] A. Capron, A. Vernes, and J. Biguet, "Le diagnostic immunoeletrophoretique de l'hydatidose," in Journees Lyonaises d'Hydatidologie, SIMEP Editions, Paris, France, 1967.

[49] D. Pagnozzi, G. Biosa, M. F. Addis, S. Mastrandrea, G. Masala, and S. Uzzau, "An easy and efficient method for native and immunoreactive Echinococcus granulosus antigen 5 enrichment from hydatid cyst fluid," PLoS ONE, vol. 9, no. 8, Article ID e104962, 2014.

[50] H. Auer, C. Stöckl, S. Suhendra, and R. Schneider, "Sensitivity and specificity of new commercial tests for the detection of specific Echinococcus antibodies," Wiener Klinische Wochenschrift, vol. 121, supplement 3, pp. 37-41, 2009.

[51] C. Lorenzo, H. B. Ferreira, K. M. Monteiro et al., "Comparative analysis of the diagnostic performance of six major Echinococcus granulosus antigens assessed in a double-blind, randomized multicenter study," Journal of Clinical Microbiology, vol. 43, no. 6, pp. 2764-2770, 2005.

[52] M. Chamekh, B. Facon, C. Dissous, A. Haque, and A. Capron, "Use of a monoclonal antibody specific for a protein epitope of Echinococcus granulosus antigen 5 in a competitive antibody radioimmunoassay for diagnosis of hydatid disease," Journal of Immunological Methods, vol. 134, no. 1, pp. 129-137, 1990.

[53] S. R. Swarna and S. C. Parija, "Dot-ELISA for evaluation of hydatid cyst wall, protoscoleces and hydatid cyst fluid antigens in the serodiagnosis of cystic echinococcosis," Revista do Instituto de Medicina Tropical de Sao Paulo, vol. 50, no. 4, pp. 233-236, 2008.

[54] S. Fotoohi, G. R. Hashemi Tabar, and H. Borji, "Serodiagnosis of human hydatidosis with an ELISA developed based on antigens derived from sheep hydatid cysts and comparison with a commercial human ELISA kit," Asian Pacific Journal of Tropical Medicine, vol. 6, no. 9, pp. 723-727, 2013.

[55] R. M. Mohamed, E. H. Abdel-Hafeez, U. S. Belal, K. Norose, and F. Aosai, "Human cystic echinococcosis in the nalut district of western libya: a clinico-epidemiological study," Tropical Medicine and Health, vol. 42, no. 4, pp. 177-184, 2014.

[56] K. Ersfeld, R. B. Gasser, and P. S. Craig, "The immunodiagnostic potential of Echinococcus granulosus adult-worm antigens in human cystic echinococcosis," Parasitology Research, vol. 83, no. 1, pp. 90-92, 1997.

[57] P. Margutti, E. Ortona, F. Delunardo et al., “Thioredoxin peroxidase from Echinococcus granulosus: a candidate to extend the antigenic panel for the immunodiagnosis of human cystic echinococcosis," Diagnostic Microbiology and Infectious Disease, vol. 60, no. 3, pp. 279-285, 2008.

[58] F. Delunardo, E. Ortona, P. Margutti et al., "Identification of a novel $19 \mathrm{kDa}$ Echinococcus granulosus antigen," Acta Tropica, vol. 113, no. 1, pp. 42-47, 2010.

[59] J. Li, W.-B. Zhang, and D. P. McManus, "Recombinant antigens for immunodiagnosis of cystic echinococcosis," Biological Procedures Online, vol. 6, no. 1, pp. 67-77, 2004.

[60] N. Ben Nouir, C. Gianinazzi, M. Gorcii et al., "Isolation and molecular characterization of recombinant Echinococcus granulosus $\mathrm{P} 29$ protein (recP29) and its assessment for the postsurgical serological follow-up of human cystic echinococcosis in young patients," Transactions of the Royal Society of Tropical Medicine \& Hygiene, vol. 103, no. 4, pp. 355-364, 2009.

[61] D. Vacirca, M. Perdicchio, E. Campisi et al., "Favourable prognostic value of antibodies anti-HSP20 in patients with cystic echinococcosis: a differential immunoproteomic approach," Parasite Immunology, vol. 33, no. 3, pp. 193-198, 2011.

[62] M. Mariconti, C. Bazzocchi, F. Tamarozzi et al., "Immunoblotting with human native antigen shows stage-related sensitivity in the serodiagnosis of hepatic cystic echinococcosis," The American Journal of Tropical Medicine and Hygiene, vol. 90, no. 1, pp. 75-79, 2014. 
[63] Y. R. Yang, P. S. Craig, A. Ito et al., "A correlative study of ultrasound with serology in an area in China co-endemic for human alveolar and cystic echinococcosis," Tropical Medicine and International Health, vol. 12, no. 5, pp. 637-646, 2007.

[64] T. Li, A. Ito, X. Chen et al., "Specific IgG responses to recombinant antigen $\mathrm{B}$ and Em18 in cystic and alveolar echinococcosis in China," Clinical and Vaccine Immunology, vol. 17, no. 3, pp. 470-475, 2010. 

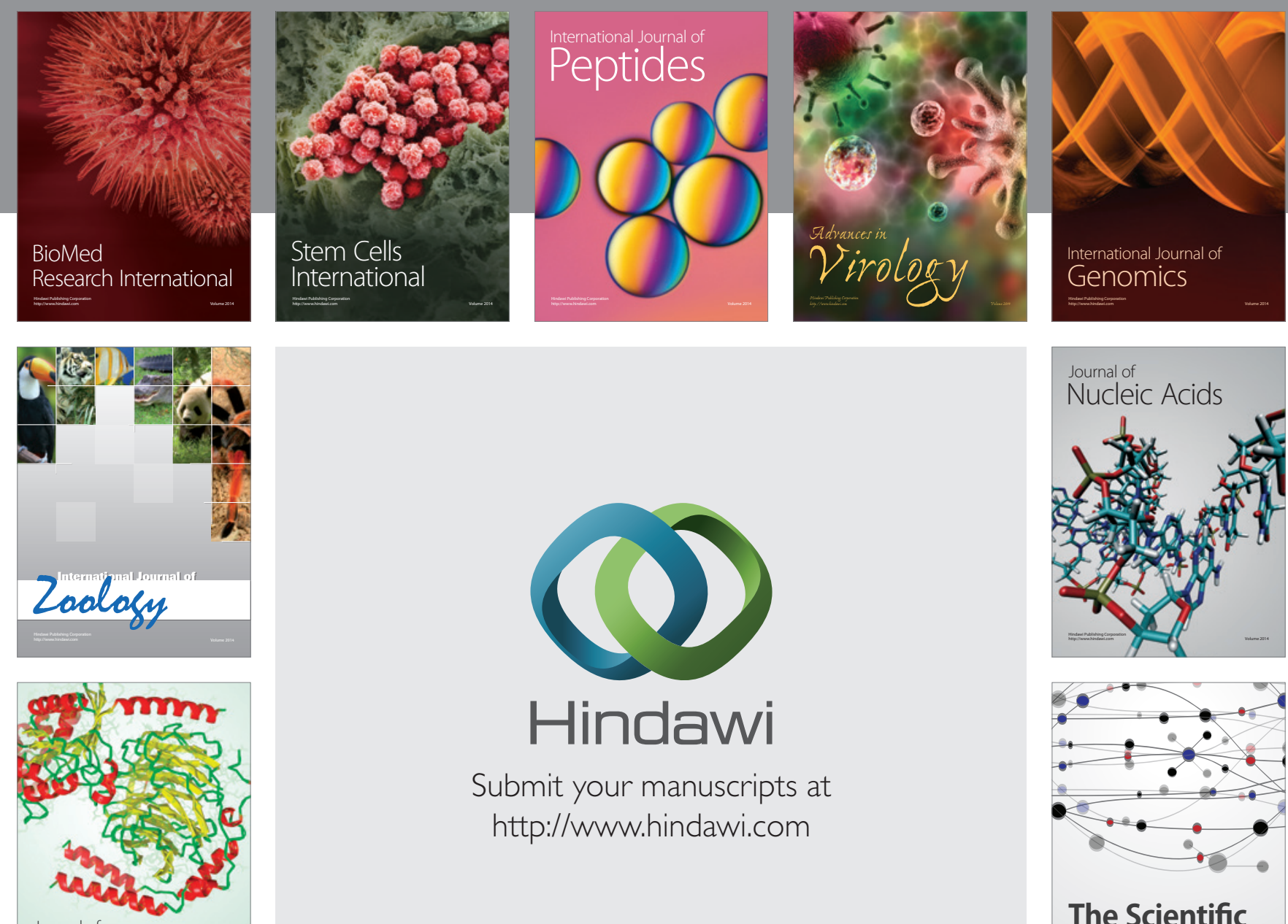

Submit your manuscripts at

http://www.hindawi.com

Journal of
Signal Transduction
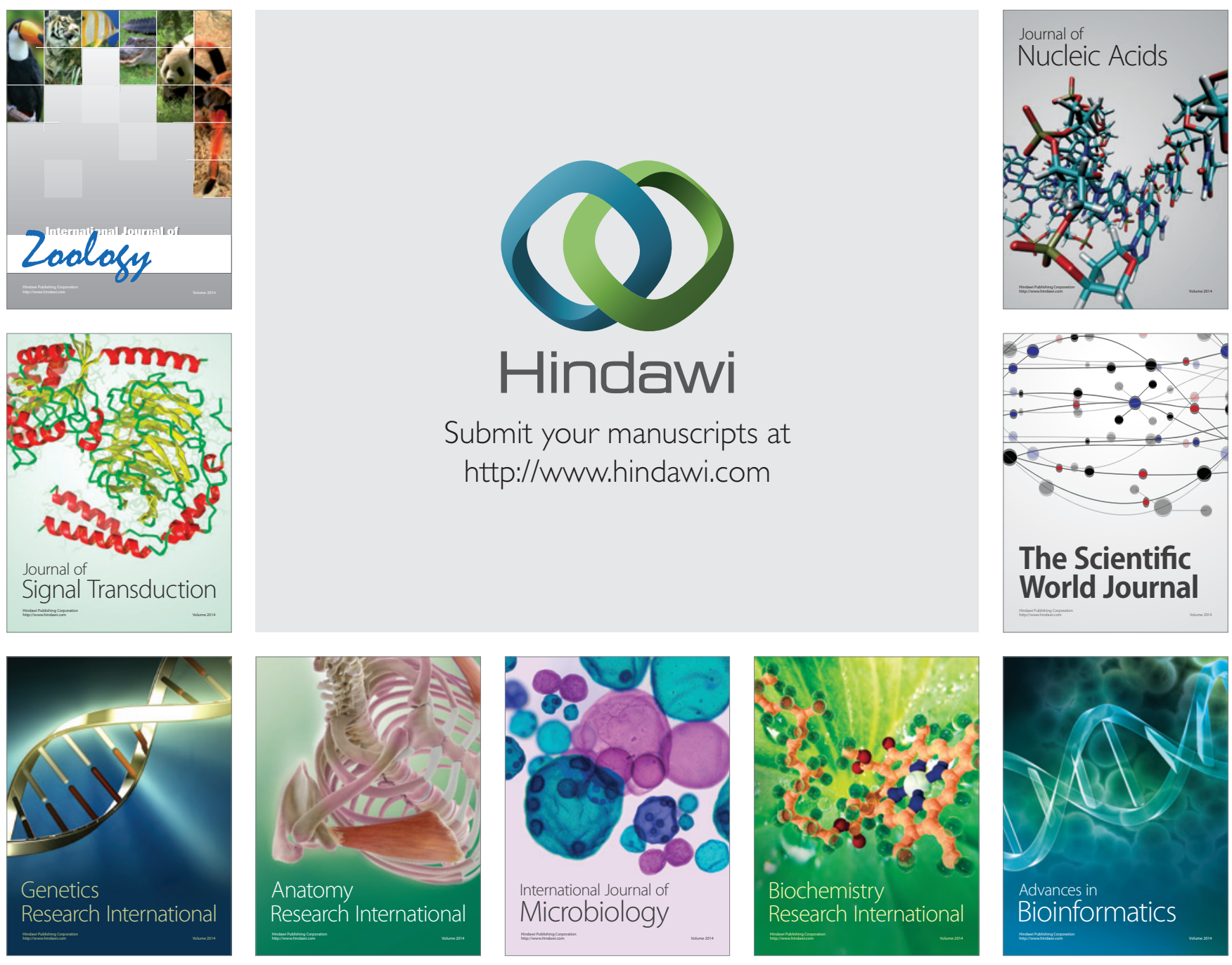

The Scientific World Journal
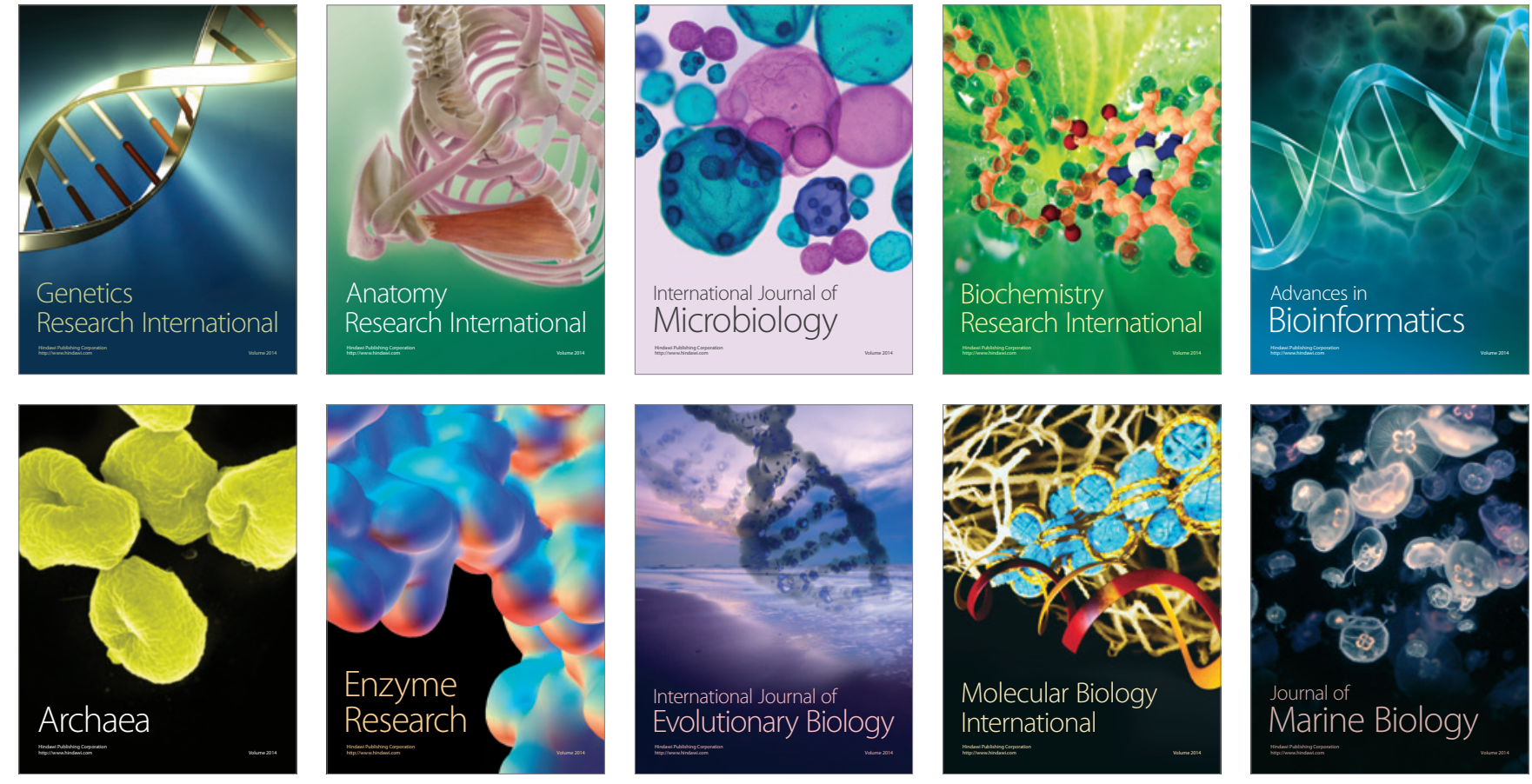